\title{
Automated Analysis of Neuronal Morphology through an Unsupervised Classification Model of Neurites
}

\author{
Amin Zehtabian', Joachim Fuchs ${ }^{2}$, Britta J. Eickholt ${ }^{2}$ and Helge Ewers ${ }^{1 *}$ \\ ${ }^{1}$ Institute for Chemistry and Biochemistry, Freie Universität Berlin, Thielallee 63, 14195 Berlin, \\ Germany \\ ${ }^{2}$ Charité - Universitätsmedizin Berlin, corporate member of Freie Universität Berlin, Humboldt- \\ Universität zu Berlin, and Berlin Institute of Health, Institute of Molecular Biology and \\ Biochemistry, Virchowweg 6, 10117 Berlin, Germany \\ * Correspondence to: \\ Prof. Dr. Helge Ewers \\ Institute for Chemistry and Biochemistry \\ Freie Universität Berlin \\ 14195 Berlin \\ Germany \\ Email: helge.ewers@fu-berlin.de
}

8

10

17 Keywords: neuronal image analysis, automatic neuron segmentation, neurite classification,

18 reconstruction, fluorescence microscopy. 


\section{Abstract}

21 Brain function emerges from a highly complex network of specialized cells that are interlinked by 22 billions of synapses. The synaptic connectivity between neurons is established between the elongated 23 processes of their axons and dendrites or, together, neurites. To establish these billions of often far24 reaching connections, cellular neurites have to grow in highly specialized, cell-type dependent patterns 25 covering often $\mathrm{mm}$ distances and connecting with thousands of other neurons. The outgrowth and 26 branching of neurites are tightly controlled during development and are a commonly used functional 27 readout of imaging in the neurosciences. Manual analysis of neuronal morphology from microscopy 28 images, however, is very time intensive and error prone. Especially fully automated segmentation and 29 classification of all neurites remain unavailable in open-source software. Here we present a standalone,

30 GUI-based software for batch-quantification of neuronal morphology in fluorescence micrographs 31 with minimal requirements for user interaction. Neurons are segmented using a Hessian-based 32 algorithm to detect thin neurite structures combined with intensity- and shape-based detection of the 33 cell body. To measure the number of branches in a neuron accurately, rather than just determining 34 branch points, neurites are classified into axon, dendrites and their branches of increasing order by their 35 length using a geodesic distance transform of the cell skeleton. The software was benchmarked against 36 a large, published dataset and reproduced the phenotype observed after manual annotation before. Our 37 tool promises greatly accelerated and improved morphometric studies of neuronal morphology by 38 allowing for consistent and automated analysis of large datasets. 


\section{Introduction}

Neuronal function manifests most obviously their complex morphology, and functional circuits require a tight regulation of the generation of each process and branching point. Dendrites serve as input structures that perform different integrations of the axonal input. Axons, on the other hand, distribute their signals to multiple cells that may be located in multiple brain regions. The connectivity patterns of neurons are both predefined in development as well as guided by local cues during neurite extension. Altered connectivity patterns in the brain seem to underly many neurodevelopmental disorders such as autism spectrum disorder, mental retardation or schizophrenia (Calhoun et al., 2012).

The complex morphology of neurons is established during development and starts to emerge during migration of newborn neurons in the developing brain. Neuronal polarization, axon elongation, dendritic arborization and synapse formation also happen in culture and the molecular players are largely conserved, making cultured hippocampal neurons an established model for the development of a functional neuronal morphology (Dotti et al., 1988; Li and Sheng, 2003; Polleux and Snider, 2010; Cembrowski and Spruston, 2019; Denoth-Lippuner and Jessberger, 2021).

The broad variety of neuronal cell types shows a wide diversity of morphological parameters that are related to function. Neuronal cell types can vary in number, length and branching of axons and complexity of dendritic arbor. The quantification of neuronal morphology via extraction of basic parameters such as neurite length, number of branches, and degree and density of branching points from microscopy images is thus of fundamental importance in the neurosciences. One of the longest used assays is Sholl analysis (Sholl, 1953), which approximates dendritic branching by counting the number of times neurites cross concentric circles emanating from the soma at increasing distances. Sholl analysis is a regular feature of neuronal segmentation software. The commonly implemented strategies range from manual tracing over semi-automatic detection (Schmitz et al., 2011) to segmentation-free analysis (Ferreira et al., 2014).

Due to their tortuous and elongated growth patterns, however, Sholl analysis is not suitable to quantify axon morphology. Axonal outgrowth has been mainly quantified by the length of the longest process, the summed length of all axonal processes, and axon complexity as the number of branch points per micron (Sainath et al., 2017; Pan et al., 2019). Extraction of these parameters require first the reconstruction of the neuronal outline and secondly the subsequent classification of processes as axons and dendrites and their primary, secondary, and tertiary branches. Commonly implemented strategies mainly use manual tracing and classifications using tools like NeuronJ (Meijering et al., 2004). 
Automated reconstruction of neurons has made significant progress over the past years, also stimulated by an interest in connectomics in projects such as the BRAIN Initiative (http://www.braininitiative.nih.gov/) or the Human Brain Project (http://www.humanbrainproject.eu/), the DIADEM challenge (http://diademchallenge.org/) or the BigNeuron project (reviewed in Parekh and Ascoli, 2013; Magliaro et al., 2019). Most current pipelines employ pre-processing strategies such as denoising and deconvolution followed by segmentation algorithms differing by the strategy they employ to distinguish neurons from background. These pipelines are implemented in commercial (Neurolucida, IMARIS, Amira, HCA-Vision) as well as free standalone packages (Neuronstudio: Rodriguez et al., 2008; Neutube: Feng et al., 2015) or as plugins in image processing software such as Matlab (TREES toolbox: Cuntz et al., 2010), ImageJ (PhD-filtering: Radojević and Meijering, 2017) or Vaa3D (APP2: Xiao and Peng, 2013; Rivulet: Liu et al., 2016; Ensemble neuron tracer: Wang et al., 2017; NeuroGPS-Tree: Quan et al., 2016; Advantra: Radojević and Meijering, 2019). An enormous number of further solutions have been also developed over the years to cope with segmentation and reconstruction of the neuron morphology, resulting in a vast number of different applications (Narro et al., 2007; Oberlaender et al., 2007; Schmitz et al., 2011; Meijering, 2010; Peng et al., 2010; Peng et al., 2014; Megjhani et al., 2015; Acciai et al., 2016; Magliaro et al., 2017; Yoon et al., 2017; Ikeno et al., 2018; Abdellah et al., 2018; Shahbazi et al., 2018; Wang et al., 2019; Abdolhoseini et al., 2019; Vidotto et al., 2019; López-Cabrera et al., 2020; Bates et al., 2020).

While these works mainly focus on the reconstruction of neurons, a reliable quantification of neuronal morphology requires the subsequent classification of neurites. Recent work describes the extraction of growth parameters (Narro et al., 2007; Scorcioni et al., 2008; Billeci et al., 2013), the modelling of these parameters to derive growth or electrophysiological characteristics (Cuntz et al., 2010; Ascoli and Krichmar, 2000) and the classification of cell types based on morphology (Armañanzas and Ascoli, 2015). These advances, however, so far do not allow for automated analysis, especially of axons, which would greatly enhance throughput in image analysis and create compatibility of this assay with highthroughput approaches.

To overcome this bottleneck and accelerate data analysis, we here developed a software that allows for batch processing of raw fluorescence micrographs of cultured neuronal cells to extract morphological parameters of axons and dendrites. We set out to use concepts and tools from the fields of automatic reconstruction and unsupervised image classification to facilitate the analysis of molecular mechanisms underlying the growth patterns of primary neurons in 2D culture. Our goal was to implement the 
102 images of neurons with a focus on developing axons where automated analysis strategies are sparse.

103 Here we present an open-source MATLAB-based software capable of classifying individual processes

104 of neurons as dendrites or axons and their branches, respectively in an automated manner and

105 benchmark it against human classification and data analysis.

106 Our software imports raw fluorescence micrographs of neurons from standard image file formats and 107 applies a set of consecutive tools for denoising, segmentation, detection of the soma, primary neurites 108 and finer, second and third order branches. This is followed by unsupervised classification of the 109 neurites. Finally, quantitative measurements are exported for each batch as a comprehensive collection 110 of figures and tables. The exported results include, but are not limited to the size of the soma, the total 111 length of the axon, number and the respective lengths of the axonal branches, dendrites and dendritic 112 branches. This information can be accessible for more specific downstream processing (Narro et al., 113 2007; Zhou et al., 2013; Gillette et al., 2015; Acciai et al., 2016; Mihaljević et al., 2018; Abdellah et 114 al., 2018).

115 We demonstrate the capability of our software on fluorescence micrographs of primary hippocampal 116 neurons and compare the achieved neurite classification with results obtained by manual image 117 analysis as ground truth. We find that the analysis of our software matches the analysis of an 118 experienced user while being capable of simultaneous analysis of tens to hundreds of images in a short 119 time without manual intervention. 


\section{Results}

\section{The GUI}

122 Here we developed a graphical user interface (GUI)-based open-source software to analyze

123 fluorescence micrographs of neurons for quantitative analysis of neurite length and branching. Files

124 are automatically imported, and visual and numeric results are saved as standard file formats for further

125 downstream processing and analysis. The main interface is shown in Figure 1. The workflow contains

126 modules for importing data, pre-processing, segmentation, neurite classification and the export of

127 quantitative measurements (see also Fig. S1).

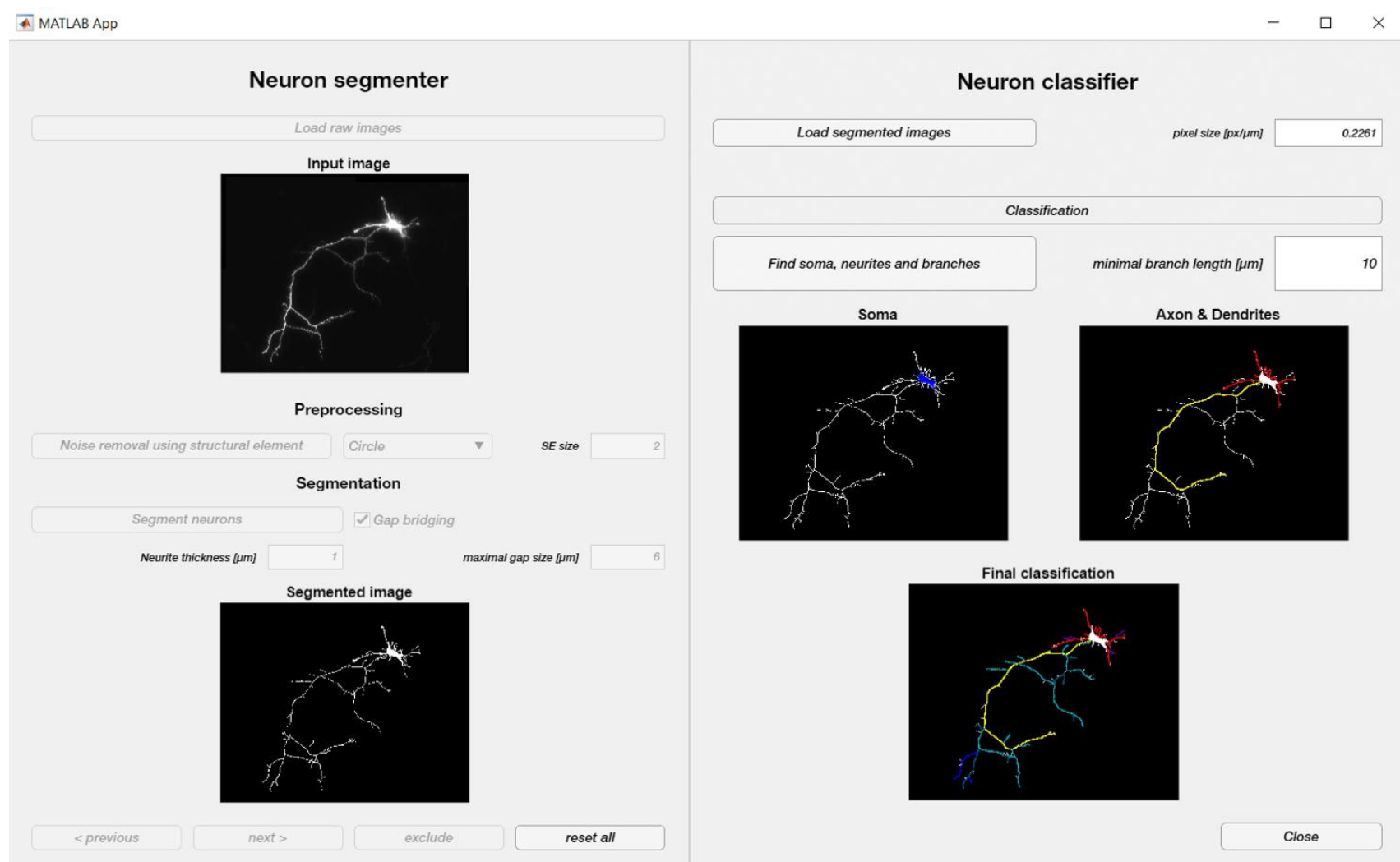

Figure 1: Graphical user interface and processing steps. Screenshot of the automated neuron reconstruction and neurite classification software's GUI. Loaded raw or preprocessed images are shown as preview (left top) and can be preprocessed.

131 The segmentation can be performed in automated or user-guided manner (left bottom). The segmented neuron is then 132 analyzed (right), the soma is detected (blue) and its neurites are classified (axon yellow, primary axon branches teal, 133 secondary axonal branches blue, dendrites red) and quantified.

134 The first block within the proposed framework allows the user to load the input data in two ways: (1)

135 loading raw neuronal images, or (2) importing data which have been segmented or skeletonized before.

136 The software can operate in two different modes. In default mode, the user can change parameter 137 settings (such as pixel size, minimal soma size, minimal neurite length) for each image individually. 
138 In batch mode, the program sequentially processes all imported data with the same settings. When the

139 batch mode is enabled, it is essential that pixel size metainformation is included in the image file.

140 After loading images, an optional pre-processing is available to smoothen the image based on two

141 different types of morphological filtering (Zehtabian and Ghassemian, 2016). Smoothing the data often

142 results in higher quality segmentation, ensuring smoother outlines of the segmented neurons and less

143 discontinuities.

144 The data will be then fed into the built-in segmentation module which follows an automated, fast

145 algorithm based on Hessian filtering. The segmented images will then be skeletonized using standard

146 techniques. If previously segmented data are loaded, they will directly proceed to the classification

147 step. The segmented neurons are then processed to automatically extract the soma, axon, dendrites,

148 axonal and dendritic branches, respectively. Quantitative measures such as the region occupied by the

149 soma, total length of the neurites as well as the length of each neurite per se will be then extracted from

150 the neurons. All numeric results and variables of interest are saved in a Matlab file and as .txt for further

151 statistical analysis. A labelled tiff image is also generated and saved for inspection of classification 152 results. 
A

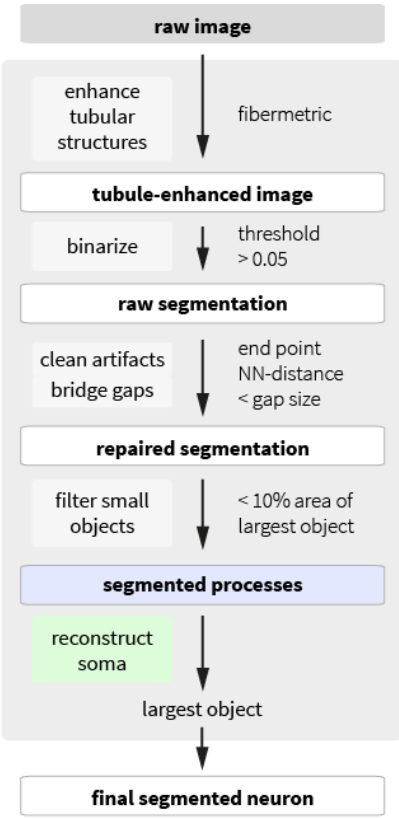

B
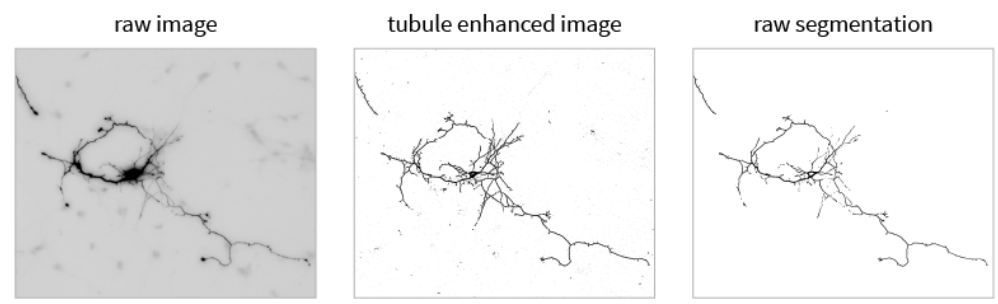

repaired segmentation
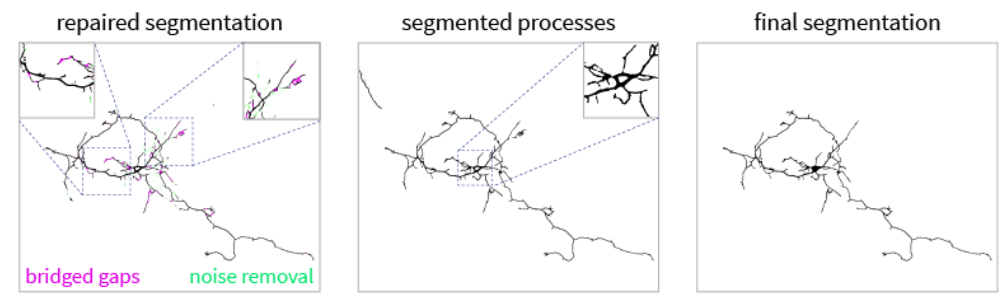

C
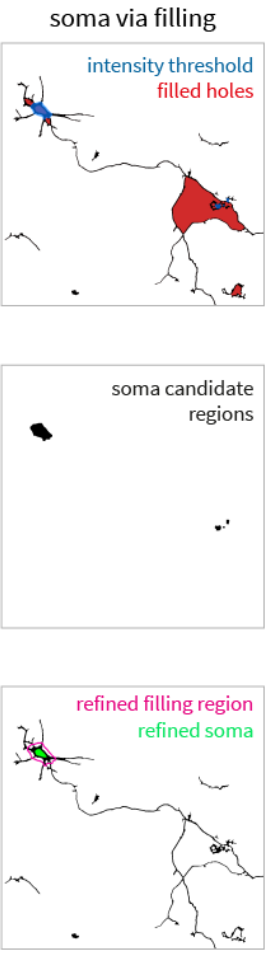

Soma reconstruction

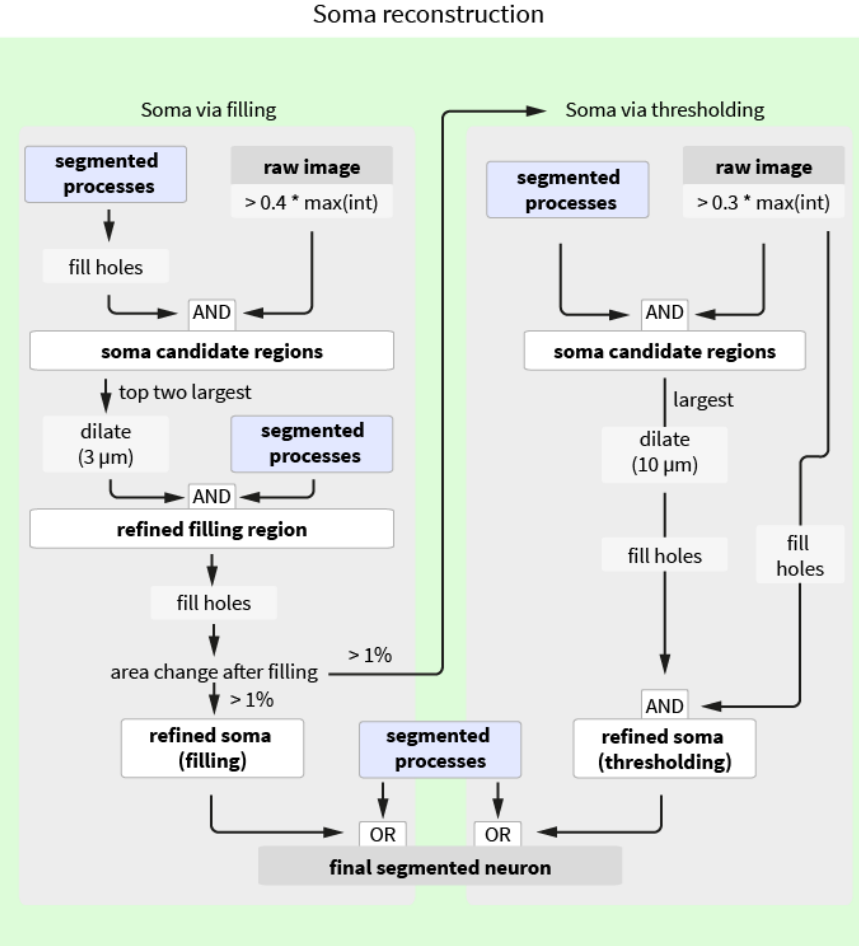

soma via thresholding

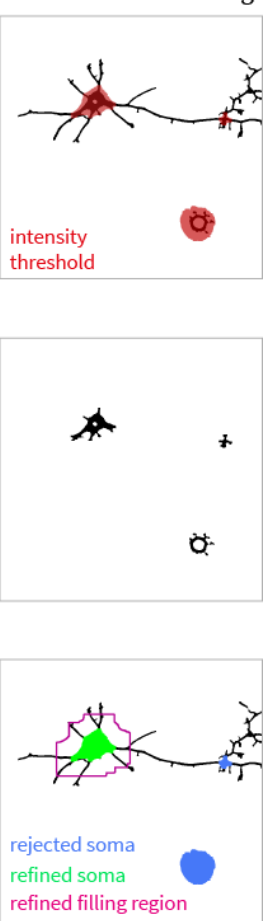

Figure 2: Schematic overview of the algorithm used in the built-in segmentation module. (A) General overview of the data processing pipeline of the proposed segmentation method. (B) Visualization of results from the individual segmentation steps performed by the proposed technique with an emphasis on quality control measures that "repair" errors in segmentation (pink and green, bottom left). (C) The soma reconstruction scheme: morphological filling serves as the preferred reconstruction method but is backed up by intensity thresholding if unsuccessful. 


\section{Segmentation}

154 One of the critical steps in morphological analysis is segmentation, i.e., the determination what is part

155 of the neurite and what is not. This can be challenging for fluorescence micrographs of neurons where

156 local differences in intensity gradients can lead to artifacts such as continuity breaks or complete loss

157 of branches. We addressed these concerns in two ways. First, we pre-processed the data to generate a

158 homogenously high quality of input images as described before, and secondly, we equipped our

159 segmentation module with multiscale filters that emphasize features in images that are important for

160 efficient neurite reconstruction. A schematic overview of the proposed neuron segmentation method is

161 depicted in Fig. 2A while example results of applying each step to a given neuron is shown in Fig. 2 B.

162 The first step of the proposed segmentation algorithm highlights elongated linear structures (which are

163 the neurites in our case). To this end, we employ a technique proposed for the detection of blood vessels

164 (Frangi et al., 1998), which is based on eigenvalue analysis of the Hessian. Hessian-based multiscale

165 filtering extracts information related to local second order structures within the image. This fibermetric algorithm enhances linear structures of a specified thickness, while minimizing information on thinner or thicker structures. In our software, the starting parameter for this 'neurite thickness' (in $\mu \mathrm{m}$ ) may be adjusted to accelerate performance of the software if prior knowledge exists. Otherwise, the software will adaptively set the value of this parameter.

170 To create the neuron model, we apply an intensity-based hard-thresholding on the tubule-enhanced

171 image to obtain a binarized image. Hard thresholding potentially introduces short gaps within the 172 neurites. This may be caused by low contrast in images or locally varying thickness of neurites, often 173 resulting in incomplete skeletonization. To overcome this problem, the software includes an optional 174 gap-filling algorithm which is based on finding and connecting nearest neighbors of endpoints from 175 unconnected objects. If the closest endpoint is closer than a maximal allowed user-specified gap size 176 (in $\mu \mathrm{m}$ ), the gap is closed with a direct line. Bridging gaps can successfully re-connect short gaps 177 between neurites (Fig. 2B, repaired segmentation left inset). However, it should be used with caution, 178 as also noise or neighboring short processes might be artificially hyperconnected when large gap sizes 179 are allowed for this algorithm (Fig. 2B, repaired segmentation right inset).

180 As can be inferred from Fig. 2B (segmented processes), although the linear structures are segmented 181 well, the interior region of the soma is not segmented. To accurately reconstruct the soma, we apply 
182 morphological operations to the binary image to fill interior image regions and holes (Fig. 2C).

183 However, individual neurites may cross paths and thus create closed-off interior regions other than the

184 soma (Fig. 2C, top left). To overcome this problem, our algorithm screens for soma candidate regions

185 (Fig. 2C, middle left) with a high average brightness. The largest two of such overlapping regions are 186 subsequently dilated and serve as region selectors for the filling operation. (Fig. 2C, bottom left). In 187 this way morphological filling is only applied around the most likely position of the soma to avoid mislocalization of the soma or overestimation of soma size.

189 While this approach works well for the majority of tested neurons, in some cases the soma region is 190 not fully enclosed after segmentation and thereby cannot be morphologically filled. Such cases are 191 detected and soma regions are then reconstructed using an intensity threshold only. To limit the effect 192 of brightness artifacts on the image, the algorithm first finds new soma candidate regions based on the 193 amount of overlap of the intensity threshold and the segmented neurite skeleton and then only 194 reconstructs the soma with largest overlap to the neuron (Fig 2C, right panels). To avoid contribution 195 of small segmentation artifacts and noise to this overlap quantification, unconnected small objects are 196 removed prior to soma reconstruction. The final step of segmentation finds the largest connected object 197 on the image and removes all unconnected objects, thereby focusing the analysis to only one cell.

198 Our software also allows for use of the automated classification only and is compatible with data 199 segmented manually in Vaa3D (Peng et al., 2010) or SynD (Schmitz et al., 2011). It is possible to load segmentations from those tools as binary image files and then to proceeding with the subsequent classification step in our software only.

\section{Classification}

204 The neurite classification scheme is illustrated in Fig. 3. The classification step initiates with the 205 detection of the soma, followed by a stepwise detection of the longest processes originating from the detected soma. The longest of these processes is classified as the "axon", the remaining processes as "dendrites". Classification proceeds by detecting processes originating from the axon as "primary 208 branches" and subsequently processes originating from these primary branches. Detection is completed 209 after no further level of branches is detected or a hard cap of maximal 10 levels of branches is reached. 210 After detection of axonal branches, the same procedure is applied to all dendrites and their branches 211 (Fig. 3). 
A

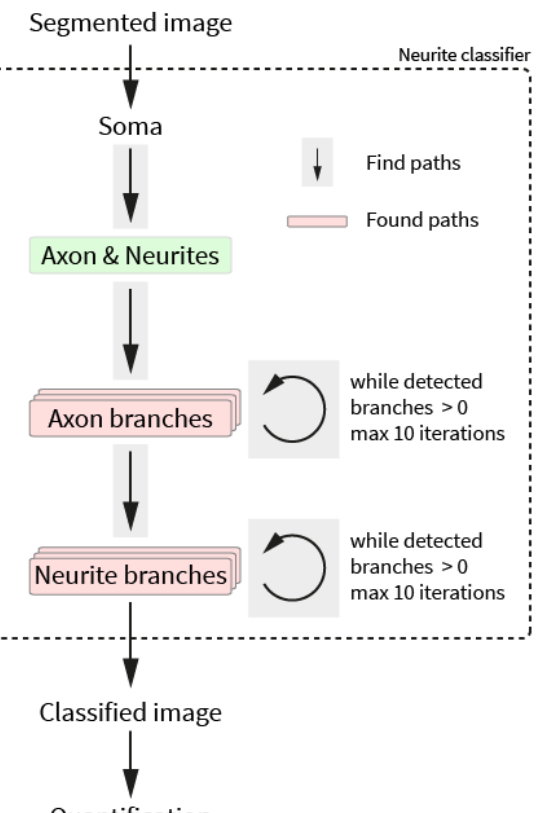

B

Find paths

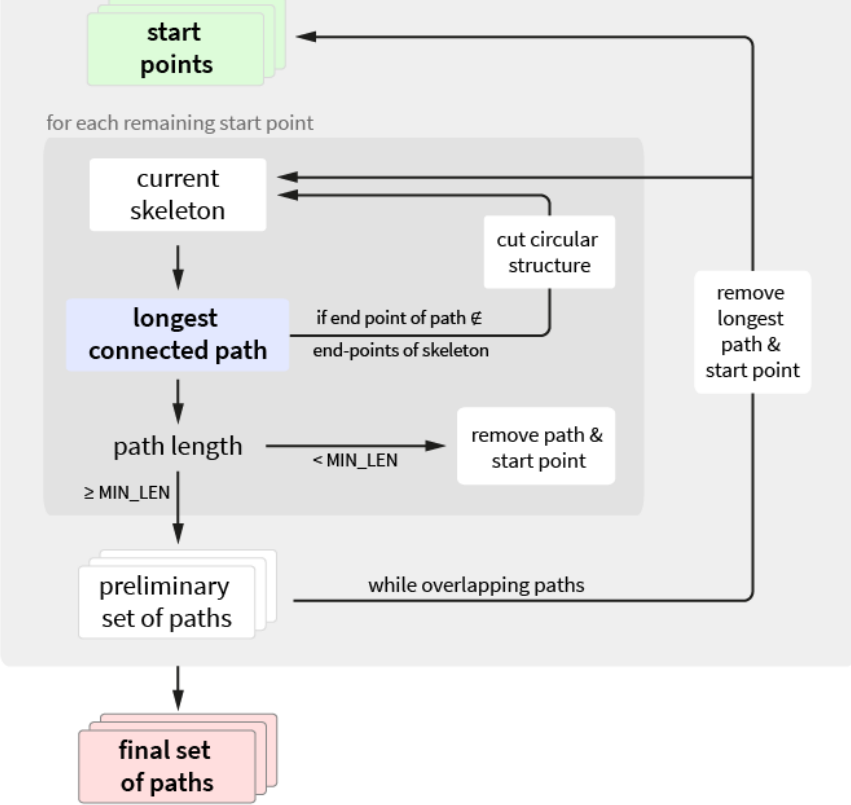

C finding longest connected path via geodesic distance transform
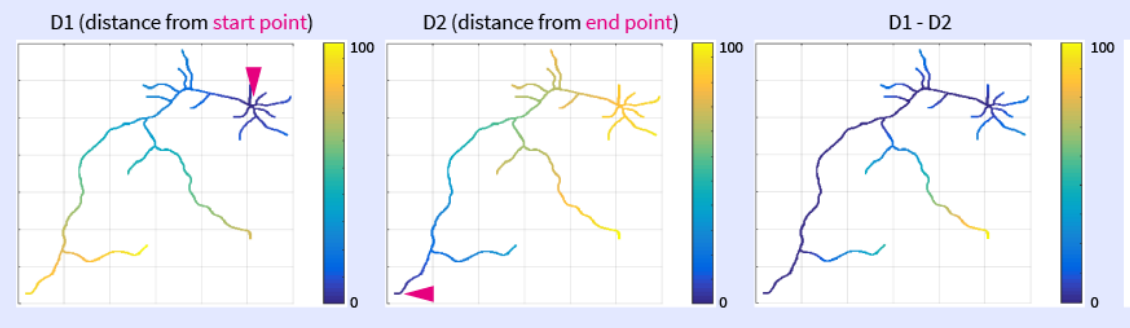

direct path: $\min (D 1-D 2)$

D

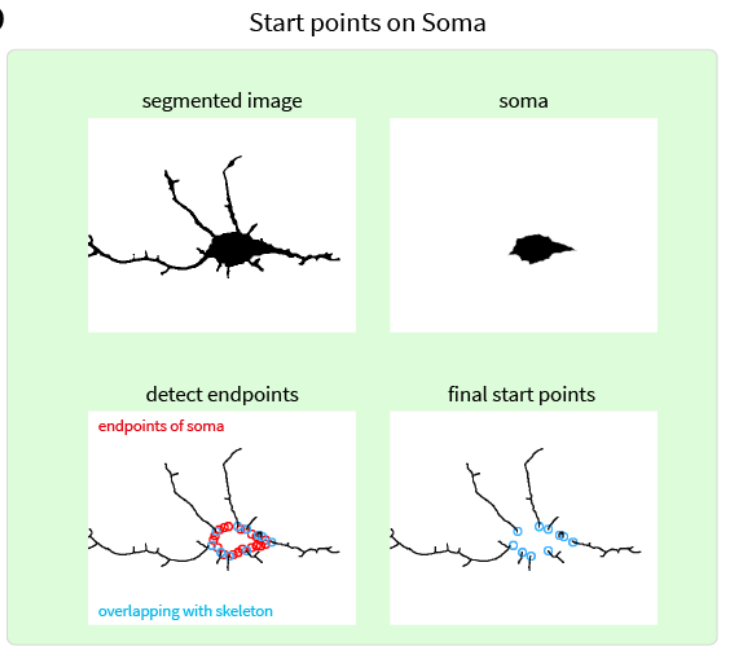

E Start points on processes

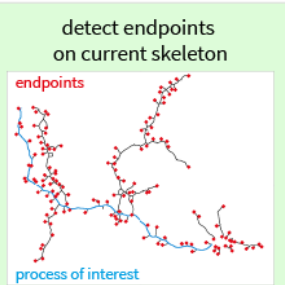

process of interest

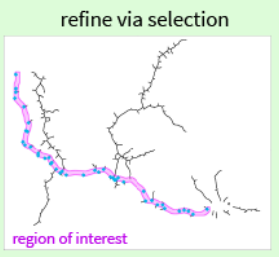

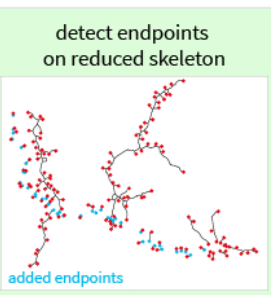

final start points

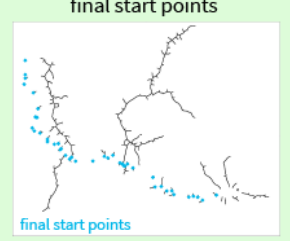

Figure 3: Illustration of neurite classification. (A) General overview of the proposed classification approach. Axon, dendrites and their branches are classified using the same algorithm 'find paths'. Axon branches are classified iteratively as primary branches, secondary, etc. before dendrite branches. (B) Strategy for 'find paths' used to detect the set of non-overlapping longest paths from a set of starting points. For each start point, the 
longest direct path in the skeleton is determined using a geodesic distance transform. In case the skeleton contains circular features precluding length measurements, these structures are separated at the originating branch point. In case a detected path does not exceed a user-specified minimal length, it is removed from further analyses. In case paths from multiple start points overlap, the longest path is stored, and all other start points are reevaluated on a skeleton without this path. (C) Determination of the longest direct connection of two points by finding the minimum of the difference of the geodesic distance transform for both points. (D-E) The reliable detection of start points defines the reliability of the classification algorithm, as paths without start points will not be classified. (D) Start points on the soma are detected as morphological end points of the soma that overlap with the skeleton excluding the soma. (E) Start points on neurites (i.e., start points for branches) are detected as the added morphological end points of the skeleton after removing the given neurite (or set of neurites) from the skeleton. To refine the selection, only points within a 5 px distance ('region of interest') to the given neurite are retained.

\section{Soma Detection}

213 Since axonal and dendritic trees both emerge from the soma, their location is required as a starting 214 point for reconstruction (Meijering, 2010). In general, even an approximation of the soma contour is 215 often difficult to be accurately reconstructed (Abdellah et al., 2018). One solution to facilitate detection 216 of the soma is to stain for DNA in a separate image channel (Meijering, 2010) to identify the soma 217 unambiguously. This does, however, not allow for an accurate measurement of soma size. In the 218 presented work we assume that neuronal images are imported to the software as single-channel data of 219 fluorophore-filled neurons.

220 Fig. 4 illustrates our proposed soma detection scheme in detail. The procedure starts by detecting the 221 largest connected component of a minimal diameter by a repeated morphological erosion operation 222 using a square (with size $l_{i} \times l_{i}$ at the $i^{\text {th }}$ step) as the structural element $S E 1$ with varying size. The $S E 1$ size follows a descending order starting from $l_{1}=S^{S E}$ (which can be set either manually or automatically). Although the erosion operation itself reduces a given structure to a slightly thinner version, since the $S E 1$ size decreases, each step of performing the repeating erosion block slightly expands the eroded image (which is called $B_{i}^{e r}$ at the $i^{\text {th }}$ step). At each step, the size of $B_{i}^{e r}$ is compared to the pre-specified minimum allowed size of the soma $S^{S o m a}$. The repeating erosion cycle continues up to the point when the size of $B_{i}^{e r}$ is no longer smaller than the threshold $S^{\text {Soma }}$. At this point, the eroded image will be checked for the number of connected components (called 'island' hereafter). In case of having multiple islands, only the largest connected component will be kept and named as $B^{l c c}$. 
231 The main soma detection step initiates with the centroid of $B^{l c c}$ found in the previous step. The centroid 232 pixel (also called 'seed point') is considered as the initial soma $\left(\operatorname{Soma}_{0}\right)$. The proposed technique then 233 carries out a local search around the seed point and expands it by adding those pixels within its 234 immediate spatial vicinity with intensities between Mean(Soma) \pm tol, in which tol is the tolerance 235 parameter.

236 The region growing step continues as long as the two following criteria are met:

$$
\text { Soma }_{i} \neq \text { Soma }_{i-1} \text { and } \quad R_{j} \geq R_{\text {thr }}
$$

237 in which

$$
\boldsymbol{R}_{\boldsymbol{j}}=\frac{\text { Size of } \text { Soma }_{\boldsymbol{j}}}{\text { Size of bounding box around } \text { Soma }_{\boldsymbol{j}}}
$$

238 and $\boldsymbol{R}_{\boldsymbol{t h} \boldsymbol{r}}$ is an upper-bound threshold for the above-mentioned ratio $\boldsymbol{R}_{\boldsymbol{j}}$ with the default value set to 0.4

239 that can be also tuned in the Software. The parameter $\boldsymbol{R}_{\boldsymbol{j}}$ is defined to cope with ambiguities in the 240 transitions from soma to dendrites (Fig. 4 - Fig. S2) .

241 We visually inspected the results of applying our proposed soma extraction method to several hundred 242 neuronal images with different shapes and morphologies and found that the reconstructed soma masks 243 delineated the real cell body in the neuron images with a high level of accuracy. 


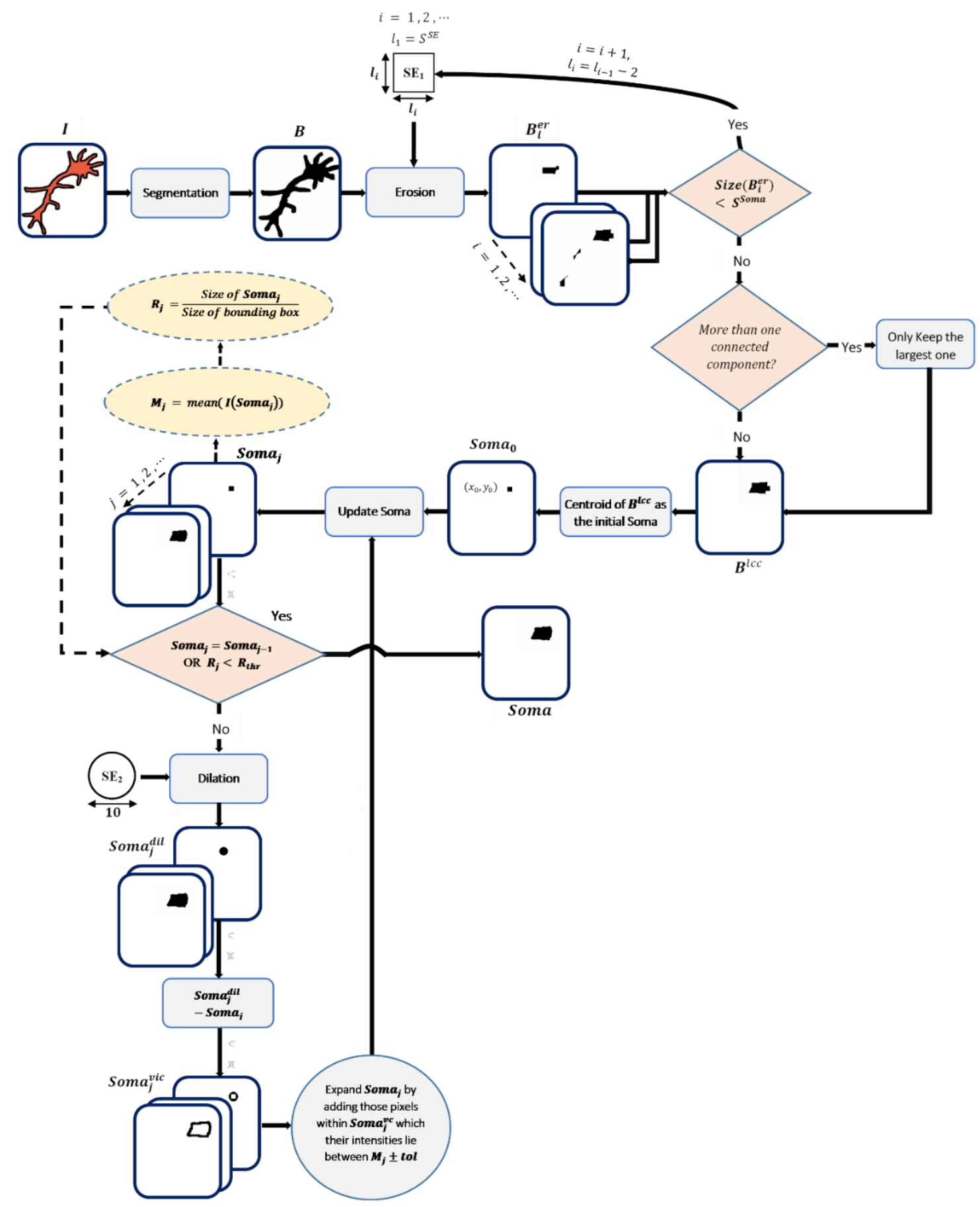

Figure 4: The soma detection scheme highlighting the individual steps in analysis.

\section{Neurite Classification}

245 The detection of the longest connected path originating from a specified set of start points lies at the

246 core of the neurite classification scheme (Fig. 3B). We here define an axon as a single process growing

247 individually for the longest possible distance from the soma. Beginning with soma detection, the

248 segmented image is transformed into a skeleton using a repetitive morphological thinning operation 
which thins the segmented foreground to its medial axis (Lam et al., 1992). Afterwards, geodesic distance transforms (Soille, 2013) starting from specified points on the skeleton are calculated (Fig. 3C, transform D1). If the maximal geodesic distance from a given start point is found on an endpoint of the skeleton, this endpoint serves as the start of a second geodesic distance transform (Fig. 4C, transform D2). The difference of D1-D2 reaches a minimum at the directly connecting path of start point and detected endpoint and is stored as a detected path.

Circular structures in the skeleton complicate the detection of a longest path, as multiple paths could be equally distant to the point of maximal distance. In such a case, the maximal geodesic distance does not coincide with an endpoint of the skeleton and the detected path would be incomplete. To overcome this problem, a repair function is initiated to trace back to where the circular structure in the skeleton starts. This is achieved by walking backwards from the point of maximal distance until the respective geodesic distance is only found once in the array of distances - representing the point where two equally long paths branch out. The repair function cuts one of the paths (randomly) by removing the first pixel after the branchpoint. Subsequently, the same starting point will be used to detect the new longest geodesic distance in the skeleton until it this distance is reached at an endpoint of the skeleton.

264 Further steps of the path-detecting algorithms mainly deal with exclusion criteria for any specific path.

265 If a detected path is below a user-defined minimal length of interest (the standard value is set to $10 \mu \mathrm{m}$

266 as a threshold to distinguish precursor protrusions from mature branches but can be modified by users depending on the specific research question), the starting point in question and the path will be deleted from the skeleton to accelerate downstream detection. Only paths longer than this maximal length are stored for further processing.

As paths from different given starting points tend to overlap if there are intersections in a skeleton, the final step deals with removing these overlaps. If overlaps are detected, the longest path and its start point in the set are saved and subsequently removed from the analyzed skeleton, and the detection is restarted with all starting points on the modified skeleton. Restarting detection for all paths has turned out to be necessary as newly detected paths on the refined skeleton have been found to often overlap with previously detected other branches, and in rare cases, more than two branches overlapped. To avoid overlap between any branches also after this re-evaluation step, this overlap-removing algorithm is repeated until no overlap is detected between any branches. The final step of the path-detection from a set of start points removes all detected paths from the skeleton for subsequent detection of higher order branches. 
The accuracy and speed of the proposed classification algorithm depends strongly on the choice of start points. For the initial step of detecting axon and dendrites, start point candidates are found as the endpoints of the detected soma. Those endpoints that overlap with the skeleton of the segmented image excluding the soma classify as final start points for axon and dendrite detection (Fig. 4D). Axon and dendrite detection then proceeds on the skeleton without the soma, to avoid detection of the axonendpoint from all possible start points (the geodesic distance for unconnected parts of a skeleton is infinite and will therefore never qualify as a path of minimal distance).

To detect start points for higher order branches on axons, dendrites or their branches, endpoints and branchpoints are detected before and after removing the originating path from the skeleton (Fig. 4E). Additional endpoints and lost branchpoints serve as candidate start points for the detection of the next order of branches. These candidates are refined by proximity to the process of interest (within $5 \mathrm{px}$ ) and the refined set will initiate the first iteration of the find paths algorithm described above (Fig. 4B).

This selection of start points on neurites is not capable of distinguishing crossing of a neurite from branching and therefore slightly overestimates the number of branches (see next section). The implemented length threshold for branch-detection nevertheless improves accuracy of detecting relevant branches when compared to simply determining branch points as pixels with more than two neighbors: morphometric branchpoint detection does not account for the length of connected processes and is therefore more susceptible to noise in images or overly detailed segmentation results - in many cases, these paths contain only individual pixels. Furthermore, detection will speed up with iterations, as parts of the skeleton that are too short to be relevant will be removed from downstream calculations.

After extracting features of interest from the classified neurites, all numeric results as well as a labelled neuron are saved in folders specified for storing data. The numeric outputs are exported to spacedelimited .txt and .csv file formats to be imported later into other software for further analysis. The segmentation and classification results are saved as .tiff files in specific folders. Additionally, all variables of interest used in the current setting will be stored in the Matlab file 'result.mat' as a global data matrix.

\section{Benchmarking}

307 In order to evaluate the performance of our proposed framework, we compared the results of our

308 algorithm with the analysis of an experienced user as ground truth. To do so, we used data from manual segmentation performed in a recent study on axonal branching (Brosig et al., 2019). 
310 This dataset consists of 883 fluorescence micrographs of neurons, 204 of which were excluded from

311 being analyzed by the software due to either the presence of large imaging artifacts, their very low

312 signal-to-noise ratio or overlapping signal from several cells. While this means that $24 \%$ of the data

313 are lost, this dataset has not been specifically acquired for automated analysis and a dedicated dataset

314 would not have required such a step. If users limit their microscopy to non-overlapping cells (also non-

315 overlapping with debris), we expect a much higher applicability. Nevertheless, we advise users to

316 quality control segmented images before proceeding with classification to improve the quality of

317 quantitative readouts. The remaining 679 images were fed into the proposed segmentation and

318 classification steps (Fig. 5A). In 2 images, the software produced a segmentation artifact precluding

319 further classification (no soma reconstructed), and in 8 images the software did not label all major

320 processes and their branches. The remaining neurons were fully segmented. 
bioRxiv preprint doi: https://doi.org/10.1101/2022.03.01.482454; this version posted March 1, 2022. The copyright holder for this preprint

A

$76 \%$ of neurons in dataset are analysable

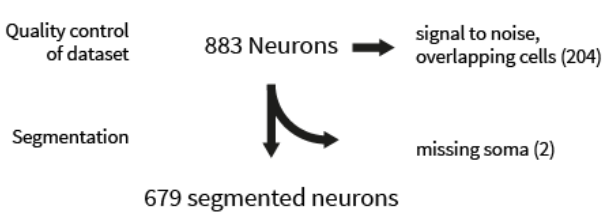

Classification

669 classified neurons

C
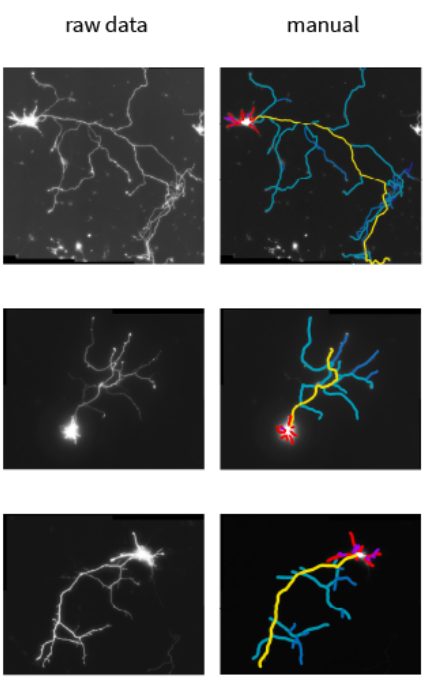

manual

software
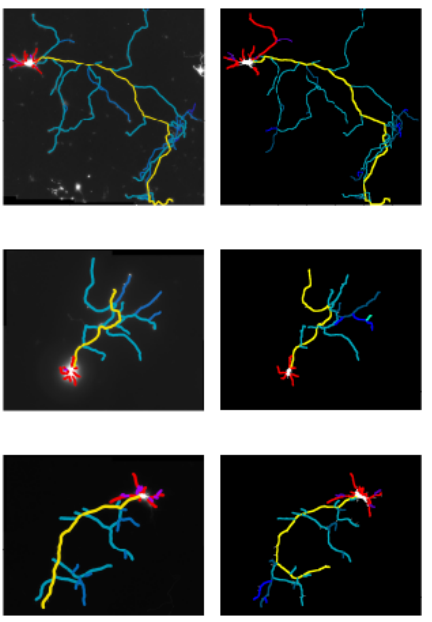

D

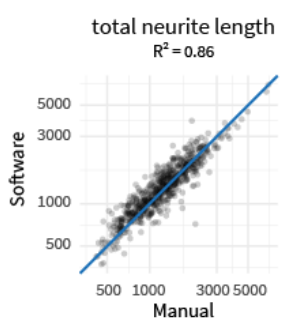

G

B

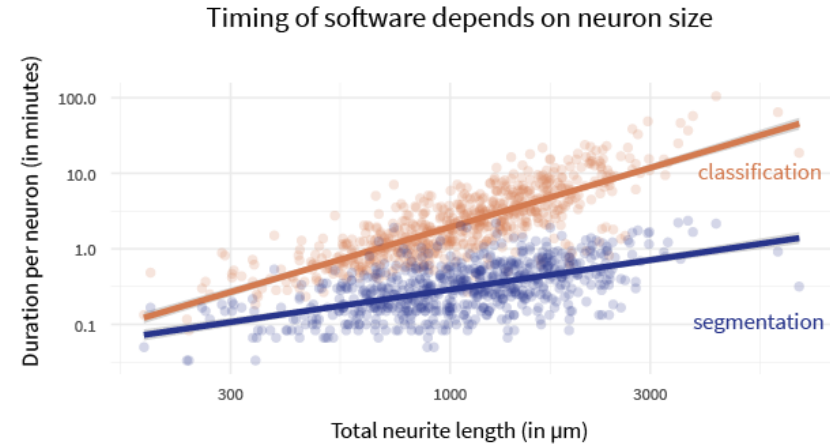

\section{E}

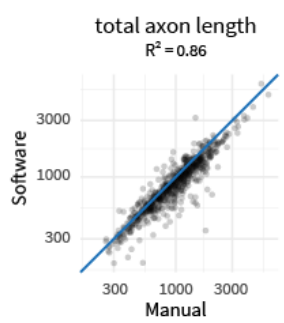

dendrite branch density
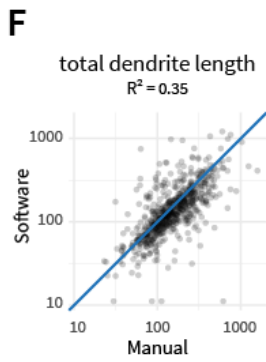

I

axon branch density $\mathrm{R}^{2}=0.5$
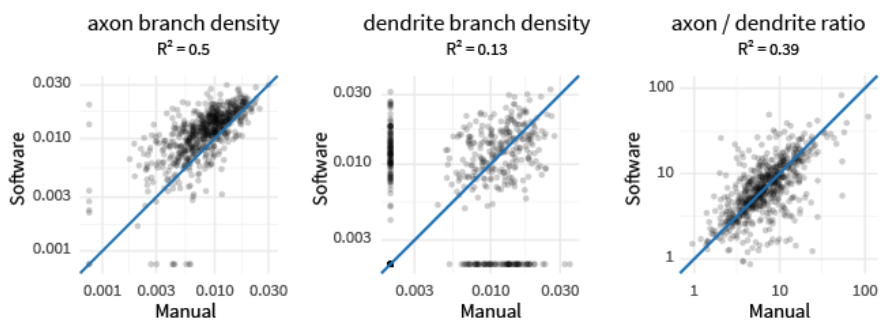
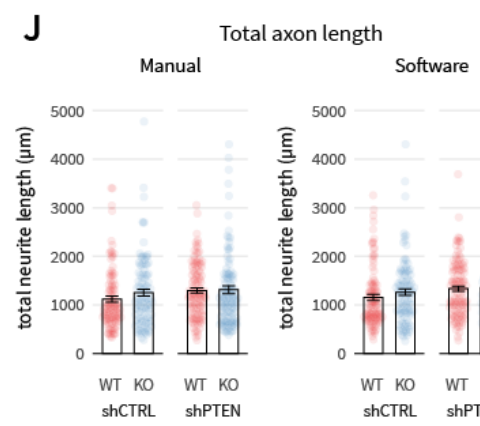

K
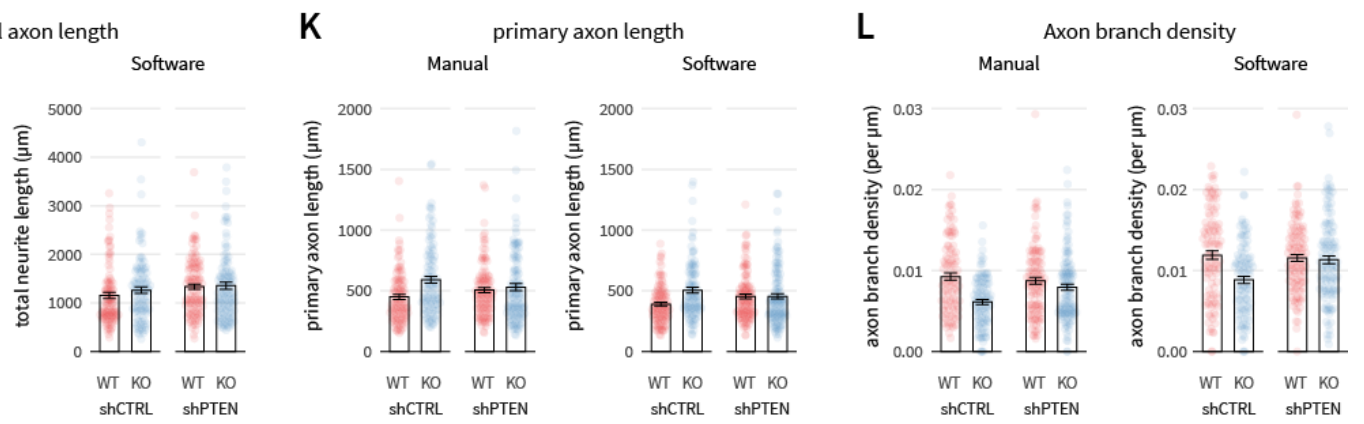

Figure 5. Benchmarking of software against a published dataset. (A) The test dataset consists of neurons manually analyzed for a study on axon branching (taken from Brosig et. al., 2019). (B) Duration of the calculation scales exponentially with neuron size. (C) Example results from the proposed neurite classification framework in comparison to expert user. (D-I) Quantitative comparisons of automated software analysis with expert user analysis. Axes in F-I are logarithmic due to the non-linear distribution of the data. Blue line and R-values are shown to visualize offset from unity between software and manual annotation. (J-K) Re-analysis of an experiment from Brosig et. al., 2019 (axon arborization in PRG2/PLPPR3-KO neurons with or without additional knockdown of PTEN). While absolute values are not identical, differences between biological treatments are reliably detected and not skewed by the software. 
321 The analyses presented were run on a laptop (Intel® Core ${ }^{\mathrm{TM}}$ i7-8565U CPU 1.80GHz, 8, 16 GB RAM,

322 Windows 10 64bit) using Matlab R2021a. Neuronal images used in our experiments are between 1.3

$323 \mathrm{MB}$ (1280 x 1024 pixels) to $29.6 \mathrm{MB}(6720 \times 4506$ pixels) in size and in 8-bit depth. The median

324 analysis time per neuron was at 2 minutes for this dataset. As shown in Fig. 5B, however, the required

325 processing time scales exponentially with image properties such as image size and neuron complexity.

326 The neurite classification is the most time-consuming step ranging between few seconds and an upper

327 border of two hours for one image. In case the desired dataset consists of larger neurons, computation

328 time could therefore increase considerably. However, our experiments show that even for large, highly

329 complex images, dozens of neurons can be batch-processed over-night in a fully-automatic manner

330 with no need for manual interventions.

331 Example results are presented in (Fig. 5C) showing the performance of the proposed method compared

332 to the manual segmentation and classification of neurites. In many cases, the software reproduces the

333 manual analysis nearly identically (Fig. 5C, top). In some cases, the expert user included additional

334 information such as neurite intensity or crossing of neurites to define the full path of an axon differently

335 (Fig. 5C, middle). In other cases, the software provided a more accurate length measurement than

336 human users and therefore chose a different endpoint for the axon (Fig. 5C bottom). In general,

337 however, reconstruction and classification of neurites appeared largely similar to expert user analysis.

338 To better evaluate the accuracy of the measurements generated by our software, we compared 339 quantitative readouts of morphology of the software to those achieved by manual analysis made by the

340 expert user (Fig. 5D-I): Total lengths of all reconstructed neurites, axon and dendrites serve as a 341 measure for neuron size, densities of axonal and dendritic branches as a measure of complexity, and 342 the ratio of axon to dendrite length as a readout for neuron polarization and therefore correct 343 classification of the axon.

344 The comparison of total length measurements reveals high levels of correlation between sizes of 345 neurons, and their axons and dendrites detected by the software and those manually reconstructed by 346 the expert (Fig. 5D-F). The neurons in this dataset were imaged at a timepoint where dendrites are not 347 fully developed and therefore still very short. Therefore, the dendrite length (Fig. 5F) as well as the 348 ratio of axon to dendrite length (Fig. 5I) vary by orders of magnitude and are presented on a logarithmic 349 scale. Nevertheless, in both readouts automated analysis correlated well with manual quantification, 350 indicating the correct automated detection of the axon. The current version of this software classifies 351 crossing points of neurites as branches, and therefore slightly overestimates branch densities (Fig. 5G- 
352 H). The axon branch density nevertheless correlates well between automated and manual analysis and 353 therefore provides a good estimate for neuron complexity. The dendrite branch density is again difficult

354 to interpret due to the short length of dendrites in this dataset with most neurons not containing any

355 branches on dendrites.

356 Despite the slight overestimation of neuron complexity, this software nevertheless is sensitive and

357 accurate enough to detect the same phenotypes between biological treatments as an expert user (Fig.

358 5J-K). The analyzed dataset has been used to establish that PRG-2/PLPPR3 knockout does not alter

359 total axon length (Fig. 5J, manual), but increases the primary axon length (Fig. 5K, manual) by

360 exhibiting less axonal branches (Fig. 5L, manual), with additional knockdown of the phosphatase

361 PTEN (shPTEN) rescuing this phenotype (compare to Fig 7C in Brosig et. al., 2019). The automated

362 analysis of the same neurons provides the same differences between treatments even though absolute

363 values of primary axon length are slightly lower and axon branch density are slightly higher compared

364 to the manual dataset. Given the fully automated implementation, we therefore believe this tool allows

365 for the faster screening of multiple biological treatments and for analyzing more neurons to achieve

366 better statistical confidence.

\section{Discussion}

369

370

371

372

373

With the introduction of large field of view cameras and automated microscopes producing large amounts of data in a short amount of time, the automated computational processing and analysis of morphometric data has become more important for neuroscience research. At the same time, automation offers great prospect in terms of data reproducibility, as experiments can be performed with many datapoints and stronger statistics, allowing for the detection of smaller effects. Furthermore, manual processing may be subject to human's biased interpretation. Reliable open-source software to extract and analyze morphometric data with minimal need for manual intervention are of interest to process a batch of neuronal images.

We present here an automated image analysis routine for segmentation, classification and quantification of the morphology of sparsely labelled, cultured neurons based on several novel image processing algorithms. The developed software offers a user-friendly GUI that lets the user interact easily with different tools which are chained together. After segmentation, our software automatically classifies all reconstructed neurites and saves a list of key output parameters to .csv and .txt file formats for consecutive analysis with statistical software. All resulting figures and illustrations are also stored 
as .tiff files within the defined output folders. The software is fully-automated with minimal need for manual intervention, enabling the analysis of hundreds of neuronal images per day.

385 For most neuronal data, the built-in segmentation module can fulfil segmentation quality expectations, eliminating the need to run any auxiliary segmentation software or tool before starting the neurite classification step. However, the accuracy achieved in by the proposed neurite classifier depends most strongly on the outcome of the segmentation step. To this end, we included optional pre-processing and gap-bridging modules to reduce the effects of noise and artifacts. For complicated cases such as multiple overlapping cells or very low signal to noise, more powerful and tunable standalone segmentation software may produce more accurate results. In such cases, instead of importing the original raw images, users can load binarized image files, which have been segmented using another software/tool, with the single requirement that the segmentation has to contain a reconstructed soma. Our software then measures total soma area and extracts information on axon, dendrite, axonal and dendritic arbors with a high accuracy. In this way, the proposed approach could be applied as a plugin or extension to open-source frameworks such as Vaa3D or commercial neuron analysis and visualization frameworks.

398 The classification module robustly detects and measures soma and neurites in $98.5 \%$ of tested cases.

399 Furthermore, it accurately reproduces relevant quantitative readouts produced in manual analysis by an expert user and is sensitive enough to detect differences between biological treatments groups. However, it does not distinguish crossing neurites from branches due to lack of three-dimensional information. This overestimates the number, and under-estimates the length of individual branches. The total length of branches is nevertheless detected accurately. One possible solution to this problem, could involve manual post-processing of the classification result. As another solution, one could define rule-based decision-making algorithms based on image features like diameter, signal intensity, curvature, or the propagation vector of the intersecting neurites in order to distinguish and label them individually.

Furthermore, due to the composition of the test dataset of early developing neurons that have elaborated axons but comparably underdeveloped and short dendrites, we were not able to objectively score the performance for mature dendrite quantification. It is possible, that dendrites might be affected more by

411 the crossing versus branching problem described above due to their proximity especially proximal to

412 the soma. Furthermore, the order of classification, with axon branches being classified before dendrite 413 branches, likely skews accuracy towards axon morphology. Users should therefore carefully monitor 414 the performance of this tool when assessing dendrite morphology and, in case phenotypes are not 
415 accurately captured, consider more dendrite-specific analysis strategies such as Sholl analysis. It is

416 conceivable, however, that the software can be readily applied to quantifying growth potential,

417 polarization, or protrusion density of neurons at earlier developmental timepoints. When used with a

418 shorter minimal length threshold and subsequent filtering by length it could also measure density of

419 branch-precursor structures such as filopodia in addition to branches individually. In the future the

420 detection of dendritic spine number, size and length could be an avenue to pursue.

421 The computational complexity of neurite branching analysis scales exponentially with neuron size. The

422 current implementation allows for a high-throughput analysis of early developmental timepoints when

423 neurons are small - without even exhausting the processor and RAM of a standard laptop. The analysis

424 can easily be run in the background while continuing working on the machine. In case larger neurons

425 are of interest, however, the duration might extend considerably, even when using more powerful

426 workstations. We nevertheless believe that the benefits of fully automated implementation outweigh

427 the downsides of analysis times and suggest batch-processing of large datasets overnight in such cases.

428 In conclusion, we present a modular, fully automated software which provides reliable segmentation,

429 classification, and quantification of the morphology of cultured neurons from two-dimensional

430 fluorescence micrographs. We have evaluated the performance of the proposed neurite segmentation

431 and classification approach by comparing it to that of an expert human user and found a high similarity

432 in reconstruction and extraction quality. The fully automated implementation of the software will

433 facilitate the quantification of large datasets containing micrographs of more neurons from multiple

434 experiments to improve statistical confidence and enable fast screening of multiple treatments and 435 thereby accelerate and improve research on neurodevelopmental mechanisms.

\section{Software Availability}

438 The software code and example raw images for testing the software are available in the software 439 repository: https://github.com/AG-Ewers/NeuronAnalysis 


\section{References}

446

447

448

449

450

451

452

453

454

455

456

457

458

459

460

461

462

463

464

465

466

467

468

469

470

471

472

473

474

475

476

477

478

479

480

481

Abdellah, M., Hernando, J., Eliemann, S., Lapere, S., Antille, N., Markram, H., and Schümann, F. (2018). NeuroMorphoVis: a collaborative framework for analysis and visualization of neuronal morphology skeletons reconstructed from microscopy stacks. Bioinformatics 34 (13), i574-i582. doi: 10.1093/bioinformatics/bty231.

Abdolhoseini, M., Kluge, M.G., Walker, F.R., Johnson, S.J. (2019). Segmentation, Tracing, and Quantification of Microglial Cells from 3D Image Stacks. Sci Rep. 9, 8557. doi:10.1038/s41598-019-44917-6

Acciai, L., Soda, P., Iannello, G. (2016). Automated Neuron Tracing Methods: An Updated Account. Neuroinformatics. 14 (4), 353-367. doi:10.1007/s12021-016-9310-0

Armañanzas, R., and Ascoli, G.A. (2015). Towards the Automatic Classification of Neurons. Trends in Neurosciences. 38 (5), 307-18. https://doi.org/10.1016/j.tins.2015.02.004.

Ascoli, G.A., and Krichmar, J.L. (2000). L-Neuron: A Modeling Tool for the Efficient Generation and Parsimonious Description of Dendritic Morphology. Neurocomputing 32-33, 1003-11. https://doi.org/10.1016/S0925-2312(00)00272-1.

Billeci, L., Magliaro, C., Pioggia, G., and Ahluwalia, A. (2013). NEuronMOrphological Analysis Tool: Open-Source Software for Quantitative Morphometrics. Frontiers in Neuroinformatics 6, 1-13. https://doi.org/10.3389/fninf.2013.00002.

Bates, A.S., Schlegel, P., Roberts, R.J.V., et al. (2020). Complete Connectomic Reconstruction of Olfactory Projection Neurons in the Fly Brain. Current Biology 30 (16), 3183-3199.e6, https://doi.org/10.1016/j.cub.2020.06.042.

Brosig, A., Fuchs, J., Ipek, F., Kroon, C., Schrötter, S., Vadhvani, M., Polyzou, A., et al. (2019). The Axonal Membrane Protein PRG2 Inhibits PTEN and Directs Growth to Branches. Cell Reports 29 (7), 2028-2040.e8. https://doi.org/10.1016/j.celrep.2019.10.039.

Calhoun, V. D., Sui, J., Kiehl, K., Turner, J., Allen, E., Pearlson, G. (2012). Exploring the Psychosis Functional Connectome: Aberrant Intrinsic Networks in Schizophrenia and Bipolar Disorder. Frontiers in Psychiatry 2, 1-13. https://doi.org/10.3389/fpsyt.2011.00075.

Cembrowski, M.S., Spruston, N. (2019). Heterogeneity within classical cell types is the rule: lessons from hippocampal pyramidal neurons. Nat Rev Neurosci 20, 193-204, https://doi.org/10.1038/s41583-019-0125-5

Cuntz, H., Forstner, F. Borst, A. Häusser, M. (2010). One Rule to Grow Them All: A General Theory of Neuronal Branching and Its Practical Application. PLoS Computational Biology 6 (8). https://doi.org/10.1371/journal.pcbi.1000877.

Denoth-Lippuner, A., Jessberger, S. (2021). Formation and integration of new neurons in the adult hippocampus. Nat Rev Neurosci. https://doi.org/10.1038/s41583-021-00433-z

Dotti, C.G., Sullivan, C.A., Banker, G.A. (1988). The Establishment of Polarity by Hippocampal Neurons in Culture. J Neurosci 8 (4), 1454-68. http://www.ncbi.nlm.nih.gov/pubmed/3282038. 
482

483

484

485

486

487

488

489

490

491

492

493

494

495

496

497

498

499

500

501

502

503

504

505

506

507

508

509

510

511

512

513

514

515

516

517

518

519

Feng, L., Zhao, T., Kim, J. (2015). NeuTube 1.0: A New Design for Efficient Neuron Reconstruction Software Based on the SWC Format. Eneuro 2 (1), ENEURO.0049-14.2014. https://doi.org/10.1523/ENEURO.0049-14.2014.

Ferreira, T.A, Blackman, A.V., Oyrer, J., Jayabal, S., Chung, A.J, Watt, A.J., Sjöström, P.J., van Meyel, D.J. (2014). Neuronal Morphometry Directly from Bitmap Images. Nature Methods 11 (10), 982-84. https://doi.org/10.1038/nmeth.3125.

Frangi, A. F., NiessenKoen, W.J., VinckenMax, L., Viergever, A. (1998). Multiscale vessel enhancement filtering. Medical Image Computing and Computer-Assisted Intervention. MICCAI'98. Springer Berlin Heidelberg. 130-137.

Gillette, T.A., Hosseini, P., Ascoli. G.A. (2015). Topological characterization of neuronal arbor morphology via sequence representation: II--global alignment. BMC Bioinformatics 16 (1), 209. doi:10.1186/s12859-015-0605-1.

Ikeno, H., Kumaraswamy, A., Kai, K., Wachtler, T., Ai, H. (2018). A Segmentation Scheme for Complex Neuronal Arbors and Application to Vibration Sensitive Neurons in the Honeybee Brain. Front Neuroinform12:61. doi:10.3389/fninf.2018.00061.

Kalil, K., and Dent E.W. (2014). Branch Management: Mechanisms of Axon Branching in the Developing Vertebrate CNS. Nature Reviews. Neuroscience 15 (1), 7-18. https://doi.org/10.1038/nrn3650.

Korobova, F., and Svitkina T. (2010). Molecular Architecture of Synaptic Actin Cytoskeleton in Hippocampal Neurons Reveals a Mechanism of Dendritic Spine Morphogenesis. Edited by Paul Forscher. Molecular Biology of the Cell 21 (1) 165-76. https://doi.org/10.1091/mbc.e09-070596.

Lam, L., Lee S.W., Suen, C.Y. (1992). Thinning Methodologies-A Comprehensive Survey. IEEE Transactions on Pattern Analysis and Machine Intelligence. 14 (9) 869 - 885. doi:10.1109/34.161346.

López-Cabrera, J.D., Hernández-Pérez, L.A., Orozco-Morales, R., Lorenzo-Ginori, J.V. (2020). New morphological features based on the Sholl analysis for automatic classification of traced neurons. J Neurosci Methods. 2020;343:108835. doi:10.1016/j.jneumeth.2020.108835.

Li, Z., Sheng, M. (2003). Some assembly required: the development of neuronal synapses. Nat Rev Mol Cell Biol 4, 833-841. https://doi.org/10.1038/nrm1242

Liu, S., Zhang, D., Liu, S., Feng, D., Peng, H., Cai, W., (2016). Rivulet: 3D Neuron Morphology Tracing with Iterative Back-Tracking. Neuroinformatics 14 (4), 387-401. https://doi.org/10.1007/s12021-016-9302-0.

Megjhani, M., Rey-Villamizar, N., Merouane, A., et al. (2015). Population-scale three-dimensional reconstruction and quantitative profiling of microglia arbors. Bioinformatics. 31 (13), 21902198. doi:10.1093/bioinformatics/btv109.

Magliaro, C., Callara, A.L., Vanello, N., Ahluwalia, A. (2017). A Manual Segmentation Tool for Three-Dimensional Neuron Datasets. Front Neuroinform. 11:36. doi:10.3389/fninf.2017.00036. 
Magliaro, C., Callara, A.L., Vanello, N., Ahluwalia, A., (2019). Gotta Trace 'em All: A Mini-Review on Tools and Procedures for Segmenting Single Neurons Toward Deciphering the Structural Connectome. Frontiers in Bioengineering and Biotechnology 7, 1-8. https://doi.org/10.3389/fbioe.2019.00202.

Meijering, E. (2010). Neuron tracing in perspective. Cytometry A. 77 (7), 693-704. doi:10.1002/cyto.a.20895.

Meijering, E., Jacob, M., Sarria, J.-C.F., Steiner, P., Hirling, H., Unser, M. (2004). Design and validation of a tool for neurite tracing and analysis in fluorescence microscopy images. Cytometry 58A, 167-176.

Mihaljević, B., Larrañaga, P., Benavides-Piccione, R., Hill, S., DeFelipe, J., Bielza, C. (2018). Towards a supervised classification of neocortical interneuron morphologies. BMC Bioinformatics. 19 (1), 511. doi:10.1186/s12859-018-2470-1.

Narro, M.L., Yang, F., Kraft, R., Wenk, C., Efrat, A., Restifo, L.L. (2007). NeuronMetrics: Software for Semi-Automated Processing of Cultured Neuron Images. Brain Research 1138 (1), 57-75. https://doi.org/10.1016/j.brainres.2006.10.094.

Oberlaender, M., Bruno, R.M., Sakmann, B., Broser, P.J. (2007). Transmitted light brightfield mosaic microscopy for three-dimensional tracing of single neuron morphology. $\mathrm{J}$ Biomed Opt. 12 (6), 064029. doi:10.1117/1.2815693.

Pan, X., Cao, Y., Stucchi, R., Hooikaas, P.J., Portegies, S., et al. (2019). MAP7D2 Localizes to the Proximal Axon and Locally Promotes Kinesin-1-Mediated Cargo Transport into the Axon. Cell Reports 26 (8), 1988-1999.e6. https://doi.org/10.1016/j.celrep.2019.01.084.

Parekh, R., Ascoli G.A. (2013). Neuronal Morphology Goes Digital: A Research Hub for Cellular and System Neuroscience. Neuron 77 (6), 1017-38. https://doi.org/10.1016/j.neuron.2013.03.008.

Peng, H., Ruan, Z., Long, F., Simpson, J.H., Myers, E.W. (2010). V3D enables real-time 3D visualization and quantitative analysis of large-scale biological image data sets. Nat Biotechnol. 28 (4), 348-353. doi:10.1038/nbt.1612.

Peng, H., Bria, A., Zhou, Z., Iannello, G., Long, F. (2014). Extensible visualization and analysis for

Polleux, F., and Snider, W. (2010). Initiating and Growing an Axon. Cold Spring Harbor Perspectives in Biology 2 (4), a001925. https://doi.org/10.1101/cshperspect.a001925. NeuroGPS-Tree: Automatic Reconstruction of Large-Scale Neuronal Populations with Dense Neurites. Nature Methods 13 (1), 51-54. https://doi.org/10.1038/nmeth.3662. 
Radojević, M., Meijering, E. (2019). Automated Neuron Reconstruction from 3D Fluorescence Microscopy Images Using Sequential Monte Carlo Estimation. Neuroinformatics 17 (3), $423-$ 42. https://doi.org/10.1007/s12021-018-9407-8.

Rodriguez, A., Ehlenberger, D.B., Dickstein, D.L., Hof, P.R., Wearne, S.L. (2008). Automated Three-Dimensional Detection and Shape Classification of Dendritic Spines from Fluorescence Microscopy Images. PLoS ONE 3 (4). https://doi.org/10.1371/journal.pone.0001997.

Sainath, R., Ketschek, A., Grandi, L., Gallo, G., (2017). CSPGs Inhibit Axon Branching by Impairing Mitochondria-Dependent Regulation of Actin Dynamics and Axonal Translation. Developmental Neurobiology 77 (4), 454-73. https://doi.org/10.1002/dneu.22420.

Schmitz, S. K., Hjorth, J. J.J., Joemai, R.M.S., Wijntjes, R., Eijgenraam, S., de Bruijn, P., et al. (2011). Automated Analysis of Neuronal Morphology, Synapse Number and Synaptic Recruitment. Journal of Neuroscience Methods 195 (2): 185-93. https://doi.org/10.1016/j.jneumeth.2010.12.011.

Scorcioni, R., Polavaram, S., Ascoli, G.A. (2008). L-Measure: A Web-Accessible Tool for the Analysis, Comparison and Search of Digital Reconstructions of Neuronal Morphologies. Nature Protocols 3 (5), 866-76. https://doi.org/10.1038/nprot.2008.51.

Shahbazi, A., Kinnison, J., Vescovi, R., (2018). Flexible Learning-Free Segmentation and Reconstruction of Neural Volumes [published correction appears in Sci Rep. 8 (1), 17585]. Sci Rep. 2018;8(1):14247. Published 2018 Sep 24. doi:10.1038/s41598-018-32628-3.

Sholl, D.A. (1953). Dendritic Organization in the Neurons of the Visual and Motor Cortices of the Cat. Journal of Anatomy 87 (4), 387-406. http://www.ncbi.nlm.nih.gov/pubmed/13117757\%0Ahttp://www.pubmedcentral.nih.gov/articler ender.fcgi?artid=PMC1244622.

Soille, P. (2013). Morphological Image Analysis: Principles and Applications. New York, NY, USA: Springer- Verlag.

Vidotto, M., De Momi, E., Gazzara, M., Mattos, L.S., Ferrigno, G., Moccia, S. (2019). FCNN-based axon segmentation for convection-enhanced delivery optimization. Int J Comput Assist Radiol Surg. 14 (3), 493-499. doi:10.1007/s11548-018-01911-z.

Wang, C.W., Lee, Y.C., Pradana, H., Zhou, Z., Peng, H. (2017). Ensemble Neuron Tracer for 3D Neuron Reconstruction. Neuroinformatics 15 (2), 185-98. https://doi.org/10.1007/s12021-0179325-1.

Wang, Y., Li, Q., Liu, L., et al. (2019). TeraVR empowers precise reconstruction of complete 3-D neuronal morphology in the whole brain. Nat Commun. 10 (1), 3474. doi:10.1038/s41467-01911443-y.

Xiao, H. and Peng, H., (2013). APP2: Automatic Tracing of 3D Neuron Morphology Based on Hierarchical Pruning of a Gray-Weighted Image Distance-Tree. Bioinformatics 29 (11), 144854. https://doi.org/10.1093/bioinformatics/btt170.

Yoon, Y.G., Dai, P., Wohlwend, J., Chang, J.B., Marblestone, A.H., Boyden, E.S. (2017). Feasibility 
bioRxiv preprint doi: https://doi.org/10.1101/2022.03.01.482454; this version posted March 1, 2022. The copyright holder for this preprint

of 3D Reconstruction of Neural Morphology Using Expansion Microscopy and Barcode-Guided Agglomeration. Front Comput Neurosci. 11:97. doi:10.3389/fncom.2017.00097.

597 Zehtabian, A., and Ghassemian, H. (2016). Automatic Object-Based Image Classification Using

598 Complex Diffusions and a New Distance Metric. IEEE Transactions on Geoscience and Remote Sensing 54 (11), 4106-4114, DOI: 10.1109/TGRS.2016.2536687.

600 Zhou, J., Lamichhane, S., Sterne, G., Ye, B., Peng, H. (2013). BIOCAT: a pattern recognition

601 platform for customizable biological image classification and annotation. BMC Bioinformatics.

602 14:291. doi:10.1186/1471-2105-14-291.

603 


\section{Supplemental Materials}

\section{Supplementary Methods:}

606 Neuron culture, microscopy and pre-processing of the validation dataset (Brosig et al., 2019)

607 Primary hippocampal neurons of either C57 BL/6 or C57 Bl/6 PRG2 -/- mice were cultured on laminin 608 and poly-ornithine coated coverslips at a density of 15,000 cells $/ \mathrm{cm}^{2}$. To visualize individual cells, 609 neurons were transfected with a plasmid encoding for green fluorescent protein (GFP) after two days

610 in culture. Neurons were fixed after five days in culture and immunolabelled for the axon marker Tau 611 and GFP. Individual neurons were imaged using a Nikon Eclipse Ti epifluorescence microscope with 612 a 40x objective (NA: 0.8). Prior to analysis, the GFP channel was converted to an 8-bit tif. Manual 613 tracing and classification of neurites was performed using NeuronJ (Meijering et al., 2004), a widely614 used ImageJ plugin based on a ridge-finding algorithm which allows semi-automated centerline tracing 615 of 2D neuron images (Narro, 2007). Neurites in the ground truth data were classified as primary axon 616 (Tau-positive and longest process originating from soma), dendrites (other processes originating from 617 soma), primary axon branches (processes longer than $10 \mu \mathrm{m}$ originating from primary axon), 618 secondary, tertiary, and quaternary branches as well as dendritic branches (originating from dendrites).

619 
621 Supplementary Figures:

622

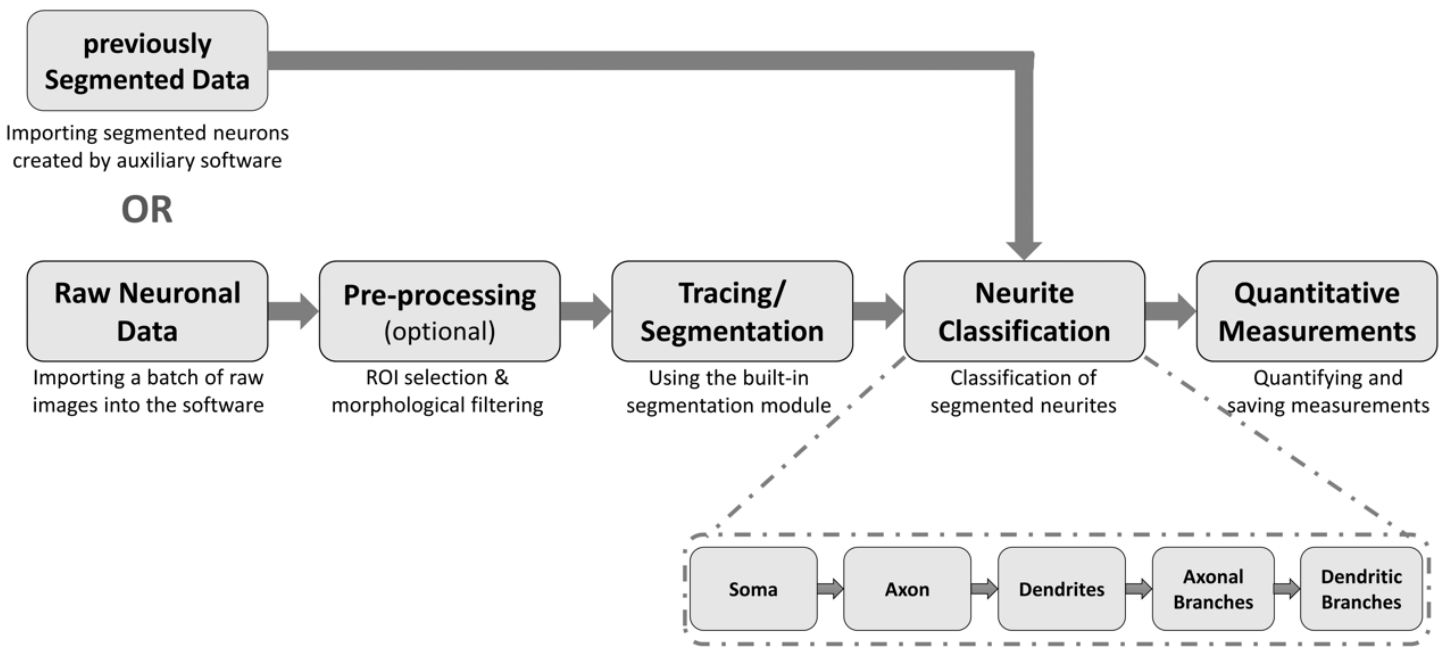

624 Figure S1: Scheme of the workflow for neuron reconstruction and neurite classification. Shown

625 are the major steps offered by the software and the respective options for the input of raw or 626 preprocessed data and the automated saving of batch processed data.

627 


\section{Short Description}

\section{Schematic Representation}

\section{A $\quad \operatorname{Soma}_{j}=\operatorname{Soma}_{j-1}$}
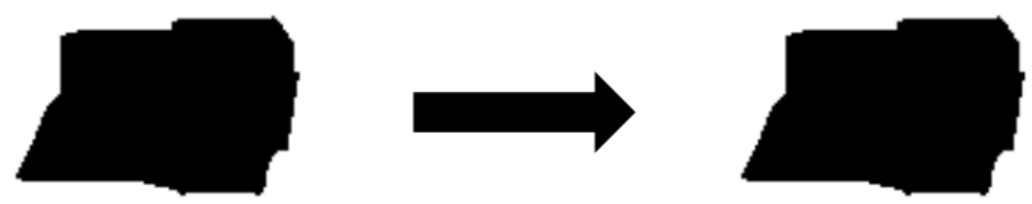

B

$$
\operatorname{Soma}_{j} \neq \text { Soma }_{j-1}
$$

$$
\boldsymbol{R}_{\boldsymbol{j}}>\boldsymbol{R}_{t h r}
$$
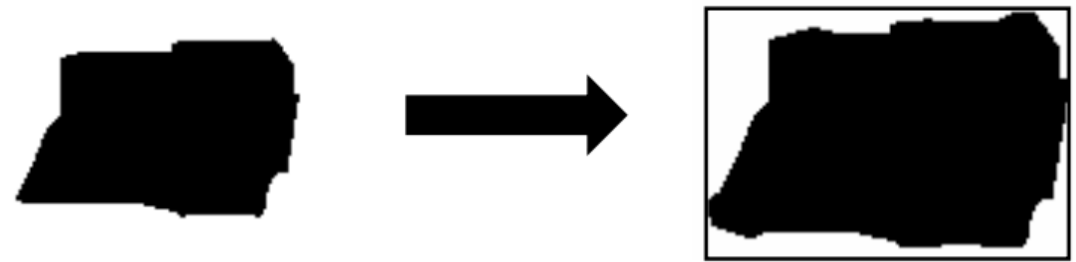

C

$$
\operatorname{Soma}_{j} \neq \text { Soma }_{j-1}
$$

$$
R_{j}<R_{t h r}
$$
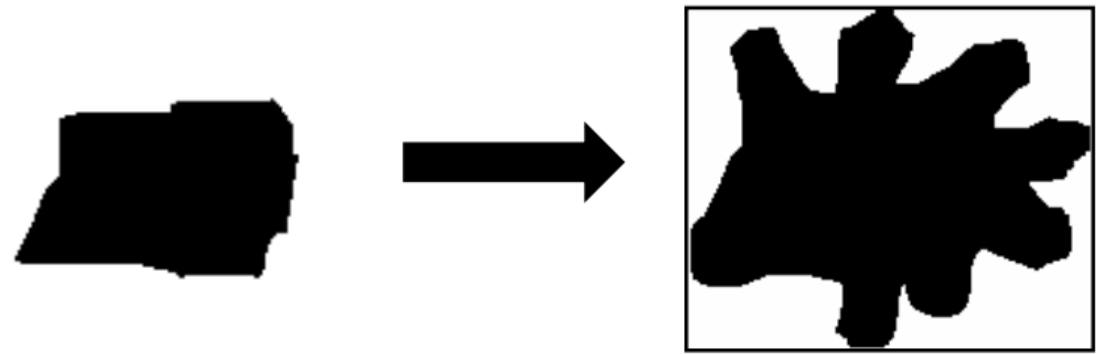

Figure S2: Different approaches for filling the soma. (A) Updating the soma does not change its size or shape anymore. This so-called 'saturated' case results in breaking the soma expansion process; (B) After analyzing the outer vicinity of the current soma, it is now expanded to cover some of the neighboring pixels as well. Since the ratio between the 'size of the updated soma' and the 'size of the bounding box' (i.e. $\boldsymbol{R}_{\boldsymbol{j}}$ ) is still lower than a pre-defined threshold (i.e. $\boldsymbol{R}_{\boldsymbol{t h r}}$ ), the expansion process will continue; (C) Similar to the previous case, the reconstructed soma is still expanding. However, the relatively low value of $\boldsymbol{R}_{\boldsymbol{j}}$ shows that the soma mask tends to overlap with some nonsoma regions, leading to transitions from soma to dendrites and/or the axon. As a result, the soma expansion should stop. 